Gynäkologische Endokrinologie 2013 .

11:241-242

DOI 10.1007/s10304-013-0560-8

Online publiziert: 30. Oktober 2013

๑) Springer-Verlag Berlin Heidelberg 2013

M. Birkhäuser

Basel, Schweiz

\title{
Geriatrische Endokrinologie
}

zeigt Alternativen zur topischen Östrogentherapie auf, die heute als wirksam empfohlen werden können. über 70 Jahren gehören Dysfunktionen der Schilddrüse einerseits und das metabolische Syndrom mit oder ohne Typ-2Diabetes andererseits zu den häufigsten endokrinen Erkrankungen überhaupt. Die direkten oder indirekten Folgen dieser und anderer nichtgonadaler Endokrinopathien für die Morbidität und Mortalität der Frau in der späteren Postmenopause werden auch heute noch oft unterschätzt, obwohl diese mit typischen postmenopausalen Veränderungen interagieren können und differenzialdiagnostisch in Betracht gezogen werden müssen. So wird bei einer älteren osteoporotischen Frau meist auf eine postmenopausale Form geschlossen, ohne die Möglichkeit einer sekundären endokrin bedingten Osteoporose zu erwägen. Nichtgonadale Endokrinopathien werden im höheren Alter leicht übersehen, weil ihre meist oligosymptomatische Klinik nicht dem typischen Bild bei einer jüngeren Frau entspricht. Bei der älteren Patientin dürfen daher unspezifische kardiale Symptome, eine zunehmende Sarkopenie oder eine Abnahme der kognitiven Fähigkeiten nicht einfach „dem Altern“ verschiedener Organe zugeschrieben werden, da sie Ausdruck einer behandelbaren hormonellen Erkrankung sein können.

Auch direkte Folgen des Östrogenmangels werden im Senium oft nicht oder nur suboptimal behandelt. Eine schwere urogenitale Atrophie mit Inkontinenz oder sexueller Dysfunktion wird von den betroffenen Frauen aus falscher Scham häufig nicht angesprochen. $\mathrm{Zu}$ den geeigneten Therapien liegen für die Frau nach 70 nur wenig zuverlässige Daten vor. K. Schiessl

\section{\) Die Folgen nichtgonadaler Endokrinopathien in der späteren Postmenopause werden oft unterschätzt}

Die Hypothyreose zeigt ebenso wie die Hyperthyreose im Alter meist ein oligooder monosymptomatisches klinisches Bild, worauf $K$. Feller und E. Christ hinweisen. Oft finden sich bei einer Hypothyreose nur unspezifischen Beschwerden wie eine Herzinsuffizienz oder Angina pectoris, eine Hypercholesterinämie oder eine kognitive Beeinträchtigung. Über eine erhöhte Fallneigung steigern Hypothyreosen das Risiko für Fragilitätsfrakturen. Auch die Hyperthyreose manifestiert sich im Alter häufig durch kardiovaskuläre Symptome (Vorhofflimmern, Herzinsuffizienz, koronare Herzerkrankung) oder durch zentralnervöse Veränderungen (Apathie, Verwirrung, Abgeschlagenheit). Im Senium kann eine Osteoporose das erste Symptom einer Hyperthyreose sein. Die typischen hypermetabolen Symptome wie Wärmeintoleranz oder Schwitzen können fehlen. Der hyperkatabole Zustand kann dennoch zu einem der senilen Sarkopenie ähnlichen Muskelschwund mit erhöhter Sturzneigung führen.

Das metabolische Syndrom betrifft rund $44 \%$ der über 70-jährigen Frauen und gehört im Senium mit oder ohne Diabetes Typ 2 zu den häufigsten endokrinen Erkrankungen. Eine von sieben 65- bis 74-Jährigen ist heute zuckerkrank.
Das anabole Insulin trägt zum Erhalt der Muskelmasse und damit zur Frakturprävention bei. Das Fehlen von oder die Resistenz auf Insulin erhöht deshalb die Fallneigung. Ein metabolisches Syndrom steigert das Risiko für eine erhöhte kardiovaskuläre Morbidität, wie dies A.O. Mueck und K. Bühling ausführen. Ein Östrogenmangel führt zu einem Blutdruckanstieg, zu ungünstigen Veränderungen im Lipid- und Glukosestoffwechsel, zu einer Zunahme von viszeralem Fett und damit zu einer erhöhten Inzidenz des metabolischen Syndroms und des Diabetes mellitus Typ 2. Dadurch steigt nach der Menopause die kardiovaskuläre Mortalität exponentiell an. Umgekehrt beeinflusst eine Östrogenbehandlung in der Postmenopause die kardiovaskulären Risikofaktoren günstig und senkt im Gegensatz zu Statinen bei der Frau die Gesamtmortalität signifikant, sofern sie innerhalb von 10 Jahren nach der Menopause oder vor dem 60. Altersjahr begonnen wird (sog. "günstiges Fenster").

Jede zweite Frau ab 50 Jahren erleidet eine osteoporotische Fraktur. Dennoch wird die Bedeutung der Osteoporose noch weit unterschätzt. Obwohl ca. 50\% der 80-Jährigen osteoporotisch sind, gaben in der Schweiz nur 19,1\% aller Frauen $>65$ Jahre an, sich wegen Osteoporose behandeln zu lassen oder deswegen in Behandlung gewesen zu sein [1]. P. Stute fasst die heutige Diagnostik und Therapie der postmenopausalen Osteoporose bei der Frau ab 70 Jahren zusammen und betont die Bedeutung des 10-JahresFrakturrisikos nach FRAX ${ }^{\odot}$ zur Bestimmung der Interventionsschwelle und des optimalen Vorgehens. Allerdings besitzt FRAX $^{\odot}$ nicht im ganzen deutschsprachi- 
gen Raum den gleichen Stellenwert. Zusätzlich zur allgemeinen Prävention [1] stehen als knochenspezifische Präparate Bisphosphonate, Strontiumralenat (mit Ausnahme der Schweiz), Denosumab und rekombinantes Parathormon zur Verfügung. Die menopausale Hormontherapie (MHT) und SERMs spielen im Senium eine untergeordnete Rolle. Wie dies in der Rubrik „Arzneimitteltherapie“ in dieser Ausgabe ausgeführt wird, empfehlen hingegen bei der früh-postmenopausalen Frau die internationalen Menopause- und Osteoporose-Gesellschaften heute innerhalb des „günstigen Fensters" die MHT als eine Behandlung der ersten Wahl [2, 3], Dies steht allerdings im Widerspruch zu den in Deutschland noch geltenden S3Richtlinien von 2009 [4].

Differenzialdiagnostisch muss bei der Osteoporose im höheren Alter immer an den relativ häufigen primären Hyperparathyreoidismus gedacht werden. I. SuterWidmer und C. Meier fassen Symptome, Diagnose und therapeutisches Vorgehen bei Hyper- und Hypoparathyreoidismus zusammen.

Zusammenfassend gilt, dass heute jeder gynäkologische Endokrinologe die Grundzüge der geriatrischen Endokrinologie kennen sollte, um seiner Patientin über 70 eine optimale Betreuung garantieren zu können. Das Übersehen nichtgonadaler Endokrinopathien führt $\mathrm{zu}$ einer erhöhten Morbidität und Mortalität.

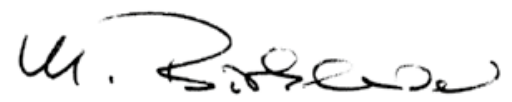

Prof. Dr. M. Birkhäuser

\section{Korrespondenzadresse}

Prof. Dr. M. Birkhäuser

Prof. emeritus für gynäkologische

Endokrinologie und Reproduktionsmedizin,

Universität Bern

Gartenstr. 67, 4052 Basel

Schweiz

martin.birkhaeuser@balcab.ch

\section{Einhaltung ethischer Richtlinien}

Interessenkonflikt. M. Birkhäuser gibt an, dass kein

Interessenkonflikt besteht.

\section{Literatur}

1. Birkhäuser M (2013) Prävention de postmenopausalen Osteoporose Teil 1: Erkennung von Risikopatientinnen und nichthormonelle präventive Maßnahmen. Gynäkologische Endokrinologie 11:129136

2. de Villiers TJ, Gass MLS, Haines CJ et al (2013) Global consensus statement on menopausal hormone therapy. CLIMACTERIC 16:203-204

3. de Villiers TJ, Pines A, Pany N. et al, on behalf of the International Menopause Society (2013) Updated 2013 International Menopause Society recommendations on menopausal hormone therapy and preventive strategies for midlife health. CLIMACTERIC 16:316-337

4. S3-Leitlinien der DGGG. Hormontherapie (HT) in der Peri- und Postmenopause (2009) AWMF-Leitlinien-Register Nr. 015/062 - Download 25.07.2013. http://www.awmf.org/leitlinien/detail/ll/015-062. html

\section{Allergische Reaktionen auf Heparine}

Bei Schwangeren besonders häufig

Während eines stationären Krankenhausaufenthalts werden zur Vorbeugung vor Blutgerinnseln häufig Heparin-Spritzen angewendet. Eine interdisziplinäre Studie zeigt nun, dass Heparin-induzierte Hautveränderungen bei fast jeder fünften schwangeren Patientin unter Heparin-Gabe auftraten.

Insgesamt wurden 111 Patientinnen in die Studie eingeschlossen. Bei 22 wurden Heparin-induzierte Hautveränderungen festgestellt. In allen Fällen war eine verzögerte Kontaktallergie die Ursache der Heparin-induzierten Hautveränderungen. Interessanterweise zeigte sich ein deutlicher Unterschied hinsichtlich der Häufigkeit der allergischen Reaktionen in Abhängigkeit vom Heparin-Präparat. Die Studie dokumentiert außerdem, dass die Heparin-Gabe besonders bei Schwangeren oft zu allergischen Reaktionen führt. Die Ergebnisse der neuen Studie tragen zu einer differenzierten und individuellen Auswahl des Heparin-Präparates bei der Behandlung des einzelnen Patienten bei. Allerdings müssen bei einer notwendigen gerinnungshemmenden Therapie mit Heparinen auf jeden Fall weitere Eigenschaften der Heparine, zum Beispiel entzündungshemmende und anti-metastatische Aktivitäten, mit in die individuelle Therapieentscheidung einbezogen werden.

Literatur: Schindewolf M, Gobst C, Kroll H et al (2013) High incidence of heparin-induced allergic delayed-type hypersensitivity reactions in pregnancy. J Allergy Clin Immunol 132:131-139

Quelle: Universität zu Lübeck, www.uni-luebeck.de 